Revista Española de Antropología Americana ISSN: 0556-6533

https://doi.org/10.5209/reaa.64974

\title{
La carga de la miseria: tiempos de crisis según los relatos históricos, proféticos y literarios mayas
}

\author{
Andrés Ciudad Ruiz ${ }^{1}$, Alfonso Lacadena García-Gallo ${ }^{2} \uparrow, \mathrm{M}^{\mathrm{a}}$ Josefa Iglesias Ponce \\ de León ${ }^{3}$ y Jesús Adánez Pavón ${ }^{4}$
}

Recibido: 20 de febrero de 2019 / Aceptado: 21 de marzo de 2019

Resumen. A partir de la interpretación de los datos proporcionados fundamentalmente por el registro arqueológico y epigráfico se han propuesto y descrito diversas situaciones y procesos de crisis y resiliencia en época maya prehispánica, analizando sus manifestaciones y tratando de inferir sus causas y consecuencias, así como, en su caso, las respuestas y estrategias seguidas por los actores implicados para su superación. Este discurso se ha realizado normalmente desde el punto de vista etic del investigador, señalando y valorando diversos marcadores como indicadores de existencia de episodios o situaciones de crisis. En este trabajo buscamos identificar las categorías que los propios mayas usaron para identificar, describir y valorar moralmente situaciones de crisis, con objeto de comparar los resultados obtenidos del análisis arqueológico y epigráfico con la evidencia emic. Para ello analizaremos las fuentes escritas mayas de época colonial, fundamentalmente textos literarios, históricos y proféticos, donde se describen épocas categorizadas como malas, míseras, de padecimiento o infelices.

Palabras clave: cultura maya; crisis políticas y sociales; resiliencia; fuentes coloniales.

\section{[en] The Burden of Misery: Times of Crisis According to Mayan Historical, Prophetic and Literary Texts}

\begin{abstract}
Based upon the interpretation of data that were essentially provided by archaeological and epigraphic records, different circumstances as well as processes of crises and resilience have been proposed during the prehispanic maya period. These circumstances and processes have been analyzed in an attempt to infer their causes and consequences as well as the responses and strategies followed by individuals who were affected and how they were able to overcome them. The circumstances and processes were usually interpreted from the researcher's ethic perspective who pinpoints and assesses different markers or signs as evidence of the existence of crisis episodes and/or calamity situations. In this contribution we attempt to distinguish the categories or indicators that the maya themselves used to identify, describe, and value crisis situations at a moral level. We then compare the results we obtained from the archaeological and epigraphic records with the emic evidence. To achieve our goal, we will analyze written maya Colonial manuscripts such as literary texts as well as historical and prophetic documents where epochs or times were labeled as bad, misery, suffering, or doomed.
\end{abstract}

1 Departamento de Historia de América y Medieval y Ciencias Historiográficas. Universidad Complutense de Madrid. andresci@ucm.es

2 Departamento de Historia de América y Medieval y Ciencias Historiográficas. Universidad Complutense de Madrid.

3 Departamento de Historia de América y Medieval y Ciencias Historiográficas. Universidad Complutense de Madrid.pepaipdl@ucm.es

4 Departamento de Historia de América y Medieval y Ciencias Historiográficas. Universidad Complutense de Madrid. adanez@ucm.es 
Keywords: Maya culture; political and social crisis; resilience; historical Colonial sources.

Sumario. 1. ¿Podemos detectar categorías mayas para definir crisis? 2. La tensión ecológica como responsable de la crisis. 3. Estados de ánimo colectivo (profecías). 4. Crisis políticas. 5. Hacia una caracterización de la crisis como categoría cultural entre los mayas de la antigüedad. 6. Referencias.

Cómo citar: Ciudad Ruiz, Andrés et al. 2019. «La carga de la miseria. Tiempos de crisis según los relatos históricos, proféticos y literarios mayas». Revista Española de Antropología Americana 49 (número especial): 283-299.

La Arqueología ha demostrado ser una disciplina de enorme valor para reconstruir el pasado humano. En estrecha colaboración con otras ciencias antropológicas y con la aplicación de un sinfín de técnicas y métodos procedentes de otras disciplinas diferentes de las antropológicas, esta reconstrucción del pasado humano ha alcanzado en las últimas décadas un grado de complejidad y de definición hasta hace poco insospechado, que se acerca al conseguido por otras ciencias que estudian al hombre, como la Historia, con la que, en cualquier caso, siempre mantiene una estrecha colaboración.

Ello no obstante, el estudio de las sociedades antiguas presenta dificultades por ahora insoslayables en algunos aspectos clave, en especial en aquellos que se refieren a su superestructura, que muestran gran dificultad y resistencia a la interpretación. En el caso maya, que es el que nos ocupa, la combinación de disciplinas científicas aplicadas en la investigación de una sociedad de larga tradición letrada, con una alta representación de asentamientos e individuos a la llegada de los españoles y una aceptada pero heterogénea continuidad cultural, algunos de estos aspectos se han podido comprender con la necesaria seguridad, mientras que otros presentan aún graves problemas de interpretación.

Uno de tales aspectos, que fue identificado desde etapas iniciales de la investigación a partir de una anomalía en el registro histórico prehispánico, y definido -si bien como concepto matizado- como colapso maya del periodo Clásico Tardío, es la comprensión de las crisis que ha sufrido este pueblo en su devenir histórico y, en cualquier caso, durante la etapa prehispánica que es la que a nosotros nos preocupa de manera particular. Si bien la arqueología, la epigrafía y la iconografía, ayudadas por una gran variedad de ciencias auxiliares, han podido detectar con bastante precisión momentos de crisis en el pasado prehispánico maya (Inomata et al. 2017), lo cierto es que no siempre hemos tenido éxito a la hora de definir con seguridad las causas que las originaron y, en numerosos aspectos, las consecuencias que tuvieron para la sociedad que las sufrió.

En diferentes reuniones del $\mathrm{GRDE}^{5}$ se han realizado propuestas de trabajo y perspectivas de interpretación de este acontecimiento histórico de profunda decadencia -y por supuesto aproximaciones teórico-metodológicas desde otras áreas- (Arnauld y Breton 2013); en este marco, el Grupo de Investigación Complutense ${ }^{6}$ del que los firmantes de este texto forman parte, ha propuesto, y ese es nuestro interés al

\footnotetext{
5 Una versión preliminar de este trabajo fue presentada en el mes de octubre de 2014 en el marco de la reunión del cuarto año del GRDE (Grupo de Investigación Europeo Past Crisis in the Americas), celebrada en la Abadia de Royaumont (Asnières-sur-Oise) y denominado «Cronología de América. Fases, cambios rápidos y crisis».

6 Investigación desarrollada por el Grupo de Investigación: «Arqueología, Historia y Etnología Maya», Universidad Complutense de Madrid (Ref.: 930518).
} 
desarrollarlo en esta ocasión, que podemos avanzar en la comprensión de las crisis que se produjeron en el área maya durante su pasado prehispánico aproximándonos a la perspectiva que los propios mayas tuvieron de estos, más o menos, dramáticos procesos a través del estudio de sus textos escritos, buscando las posibles referencias y descripciones que sus protagonistas hicieron de las situaciones y su valoración. Si podemos acercarnos al pensamiento maya acerca de lo que son, lo que significan y por medio de qué categorías se explican sus crisis - cuándo y cómo se originaron, por qué y qué consecuencias tuvieron-, podremos quizá abrir otra vía para acercarnos a la comprensión de estos fenómenos del pasado que, hasta el día de hoy, y al menos en lo que se refiere a procesos tales como el colapso del Preclásico y del Clásico y el hiato, entre otros, han resultado ser tan esquivos para la investigación.

\section{1. ¿Podemos detectar categorías mayas para definir crisis?}

Una cuestión previa que hemos de plantearnos es si, a partir del rastreo de los documentos escritos durante la etapa prehispánica y en los primeros siglos de la colonia, somos capaces de identificar categorías que se centren en las angustias y las crisis, individuales y/o colectivas, que aquejaron a los mayas.

¿Qué le preocupaba al maya de la etapa prehispánica? ¿qué hechos le provocaban angustia, y hasta qué punto estos acontecimientos afectaban al individuo o a la comunidad? Los documentos son claros y explícitos: al maya, como individuo o como colectivo, le inquietaban las mismas cosas que al conjunto de sociedades humanas, colocadas cada una de ellas en su contexto espacial y temporal. Y se refirió a estas situaciones críticas por medio de expresiones que son reiterativas en sus textos; por ejemplo, «No será malo», «No malo», «Era bueno todo», «Se perderá», «Tiempo loco», «Tristeza», «Miseria», «Padecimiento», «Martirizaba», «Infelices», «Pobrecitos», «Anticristo», «Tigre de los pueblos», «Gato montés de los pueblos», «Chupador del pobre indio», «Lágrimas de sus ojos», «Dispersión», «Fue abandonada», «Fue arruinada», «Fueron abatidos», «Medido estaba», «Sabiduría», «Saludables», «Pecado», «Enfermedad», «Rectamente erguido», «Miedo», «Marchitar las flores», «Sorber las flores», «trono de dos días», «Abrasador», «Frescura», «Como animales», «Hambre», «Ponzoña de la peste», «Abandonaron», «Establecidos», «Bajo los árboles», «Bajo las cenizas», «Fueron dispersados», «Bosques desiertos», «Vencidos», «Confusión»..., entre otros más.

En las páginas siguientes vamos a analizar dos de estas categorías de crisis, las producidas por problemas ecológico-económicos y las referidas a las crisis políticas.

\section{La tensión ecológica como responsable de la crisis}

Los mayas forman parte de una civilización agrícola a cuyo origen y desarrollo hicieron una decidida aportación; el éxito obtenido a través de esta concluyente contribución les permitió obtener un complejo diseño cultural de gran eficacia para su pervivencia y evolución por más de dos milenios. Pero a la vez esta sociedad fue consciente de que esta decidida apuesta presentaba ciertas debilidades que podían poner en riesgo, cuando no abortar, su forma de vida y el éxito cultural laboriosamente construido por largo tiempo: una vulnerabilidad básica deriva de su peculiar 
sistema sociopolítico, pero otra no menos importante es la naturaleza del clima que afectó a la región y el singular ecosistema de los bosques tropicales.

En los documentos mayas es recurrente la preocupación por la sequía; temor permanente que parece impregnar la mentalidad indígena y se convierte en el tema reiterado de múltiples profecías que relacionan los malos tiempos, los tiempos de crisis, con temporadas concatenadas de sequía. Hoy día, quizá muy preocupados por las consecuencias que puede acarrear el, para muchos científicos más que constatado, «cambio climático» en el que ya nos vemos inmersos, muchos arqueólogos y científicos que estudian el denominado colapso maya del Clásico apuestan por la existencia de fuertes episodios de sequía para explicar este fenómeno (Hodell et al. 1995; Gill 2000; Iannone 2014; Douglas et al. 2015). Esta preocupación es explícita en muchos textos indígenas.

Así, el Chilam Balam de Chumayel insiste en su narrativa con pasajes que informan o anuncian la llegada de

«Un tiempo abrasador, después un tiempo de frescura» (Bricker 1990: 93),

«Su cielo estará cargado de rayos. Sin lluvias chorreará el pan del katún. Multitudes de lunares son la carga del Katún» (Bricker 1990: 140);

o, la más dramática:

«Ocho Ahau es el nombre del Katún... «¡Ha crecido nuestro dios!», decían sus sacerdotes (...) «He aquí que vienen abundantes soles», decían. Y ardieron las pezuñas de los animales, y ardió la orilla del mar. «¡Éste es el mar de la amargura!»... Y fue mordido el rostro del Sol. Y se oscureció y se apagó su rostro. Y entonces se espantaron arriba. « Se ha quemado!, ¡ha muerto nuestro dios!», decían sus sacerdotes» (Libro de Chilam Balam de Chumayel 1985: 49).

La sequía pertinaz y prolongada, y la consecuente hambruna que genera, se instala de manera permanente en las preocupaciones de los mayas de Yucatán, quienes hacen referencia continua a ella. Podemos notar que la consecuencia de la sequía es el hambre de la población, pero el propio texto insiste en que «ha muerto nuestro dios», como si todo el sistema mental maya estuviera ligado al éxito alimentario y los propios dioses dependieran de él, de la abundancia: el compromiso de la divinidad con la abundancia queda así sellado en la mentalidad maya; y en consecuencia de él depende su éxito como comunidad humana. La sequía y la falta de abastecimiento originan, también, etapas de sacrificio y dolor extremos, tal y como se describe en los Chilam Balam de Chumayel:

«Se comerán árboles, se comerán piedras, se perderá todo sustento dentro del Once Ahau Katun» (Libro de Chilam Balam de Chumayel 1985: 50).

«Hubo hambre los cinco años, de 1650, 1651, 1652, 1653 y 1654 años» (Bricker 1990: 123).

«En tiempo del Cuatro Ahau Katun, Padres, como hormigas irán los hombres detrás de su sustento; porque como fieras del monte estarán hambrientos, y como gavilanes estarán hambrientos, y comerán hormigas y tordos, y grajos, y cuervos, y ratas» (Bricker 1990: 183).

Sequía y hambruna son, pues, dos de las preocupaciones que con insistencia aparecen en los textos mayas coloniales. Pero no se trata de un temor existente sólo durante el periodo Colonial. En los códices mayas posclásicos aparece claramente 
la mención a los periodos de ha'al «lluvia»y a los augurios buenos en los que habrá oox wi'il «abundancia de comida», wi'il uk'ul «comida (y) bebida» (Figura 1); los augurios malos son los tiempos de k'intuun «sequía» y kimlal «mortandad». Es ciertamente interesante que nos podamos remontar todavía más atrás en el tiempo, al periodo Clásico, y documentar las primeras referencias detactadas que mencionan estas calamidades: en el año 764 d.C., un sacerdote de Comalcalco, Ajpakal Tahn, inscribió o mandó inscribir un breve texto sobre una espina de raya de sacrificio posiblemente usada en un ritual asociado de adivinación- (Armijo 1999; Armijo y Zender 1999; Armijo et al. 2000; Zender 2004: 250), preguntando si wa 'iij k'intuun, wa'iij wi'naal tu' uuxlaju'n tuun «habrá sequía, habrá hambruna en el decimotercer tuun» (Figura 2).

Las percepciones positivas de la experiencia pasada o de la futura realidad son vistas, por contraste, como tiempos de abundancia:

«Con siete tiempos de abundancia se asienta el Katun, el Cuarto Ahau Katun en Chichén. Siete tiempos de abundancia son el asiento del Gran derramador de agua. Tapado está su rostro y cerrados sus ojos bajo sus lluvias, sobre su maíz abundantemente derramado. Llenos de hartura están su estera y su trono. Y se derrama su carga. (...) sea aplastado por el chorro del pan del Katun» (Bricker 1990: 139).

«Llegarán plumajes, llegarán pájaros verdes, llegarán fardos, llegarán faisanes, llegarán tapires; se cubrirá de tributo Chichén» (Bricker 1990: 139).
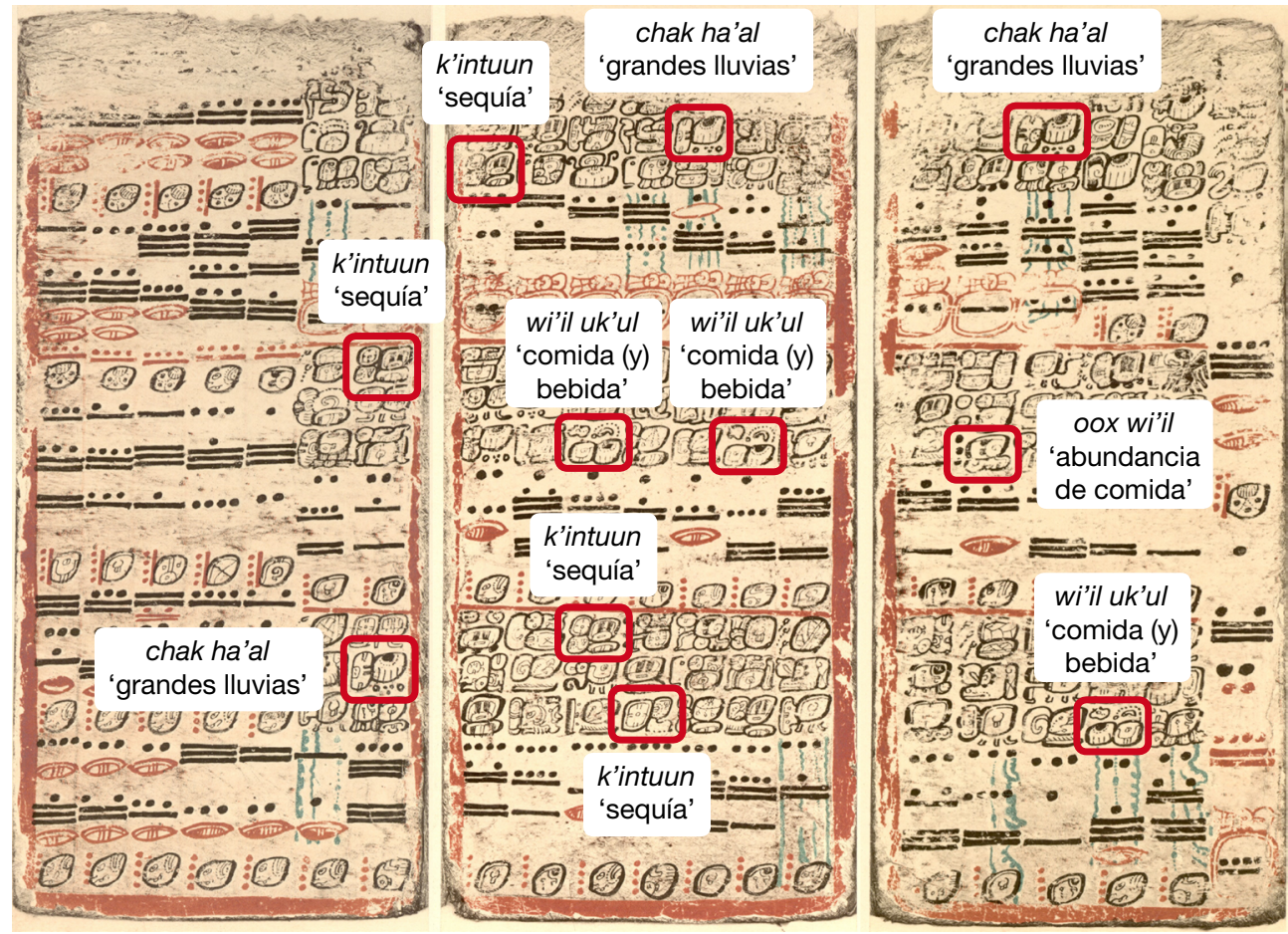

Figura 1. Textos de las páginas 71 a 73 del Códice de Dresde (copia digital de la edición de Ernst Förstemann, http://www.famsi.org/mayawriting/codices/pdf/ dresden_fors_schele_all.pdf). 


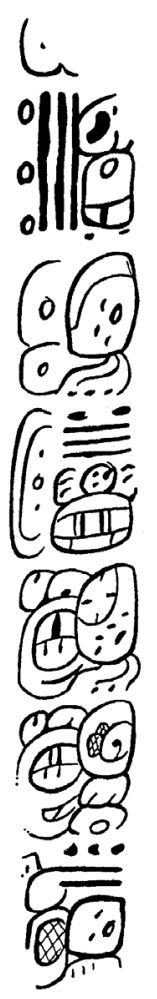

Figura 2. Texto de la Espina 3, Urna 26, de Comalcalco datada en el año 764 d.C.: wa'iij k'intuun, wa'iij wi'naal tu'uuxlaju'n tuun, «habrá sequía, habrá hambruna en el decimotercer tuun» (Zender 2004: fig. 73).

La sequía, la hambruna que provoca, la desesperación de la comunidad agrícola, traen como consecuencia el desorden social, la competición por la producción, la migración y la guerra:

«El Diez Ahau Katun, Chablé es el asiento del Katún. Allí llegarán sus pobladores. Y los árboles del bosque se doblarán sobre ellos, que serán los reyes de la tierra. Arderán las pezuñas de los animales; arderán las arenas del mar; arderán los nidos de los pájaros. Reventarán las cisternas. Grandes sequías son la carga del Katún. Es la palabra de Nuestro Padre Dios y de la Señora del Cielo [U Colel caan]. Nadie podrá escapar al filo de la guerra. Es la palabra de Nuestro Padre Dios, Dios Hijo, Señor del Cielo y de la Tierra. No ha pasado. Va a suceder con todo rigor sobre todos» (Bricker 1990: 172).

Es interesante y, por lo que estamos viendo, probablemente no casual, que en una serie de augurios asociados a un año adverso en el Códice de Dresde (Figura 3), se enumeren juntos «jaguar blanco» (una posible referencia a depredación y bestialidad), «pedernal» (posible alusión a la guerra) y «sequía»:

sak Hix haab'il, tok' haab'il, k'intuun haab'il u kuch

«tiempo del jaguar blanco, tiempo de pedernal, tiempo de sequía es su carga» (Códice de Dresde 1888/1892: 26).

Debemos buscar, entonces, en nuestras investigaciones arqueológicas no sólo las evidencias de catástrofes naturales como causa desencadenante de una crisis, sino también las consecuencias que traían aparejadas de desorden y competición por los recursos escasos, incluso la intervención oportunista de un reino limítrofe que saca partido de la situación de debilidad de otro. Quizá una vía interesante es la propuesta recientemente por Jobbová et al. (2018), quienes analizan el cambio en el comportamiento ritual maya a lo largo del Clásico Terminal y lo sostienen hasta los tiempos coloniales. Para estos investigadores los grandes rituales públicos de la elite maya se desplazaron desde un foco primordial centrado en los nacimientos, entronizaciones, matrimonios, guerras y rituales de la realeza, a aquellos que tuvieron un fundamento profundo en la fertilidad y la abundacia de los alimentos. Es muy posible que este desplazamiento del comportamiento ritual esté estrechamente vinculado con cambios severos en el limitado régimen de precipitaciones que acarrearon sequías recurrentes durante todo el periodo Postclásico; una situación paleoclimática que ha sido consistentemente contrastada por la investigación reciente (Curtis et al. 1996; Kenneth et al. 2012; Iannone 2014; Douglas, Brenner y Curtis 2015).

\section{Estados de ánimo colectivo (profecías)}

La evidencia epigráfica disponible desde el periodo Clásico indica una fuerte presencia ideológica de la religión en la sociedad maya. Nuestra percepción del Postclási- 


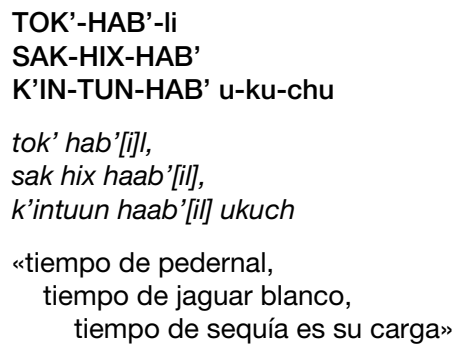

Figura 3. Texto de la página 26 del Códice de Dresde (copia digital de la edición de Ernst Förstemann, http://www.famsi.org/mayawriting/codices/pdf/ dresden_fors_schele_all.pdf).

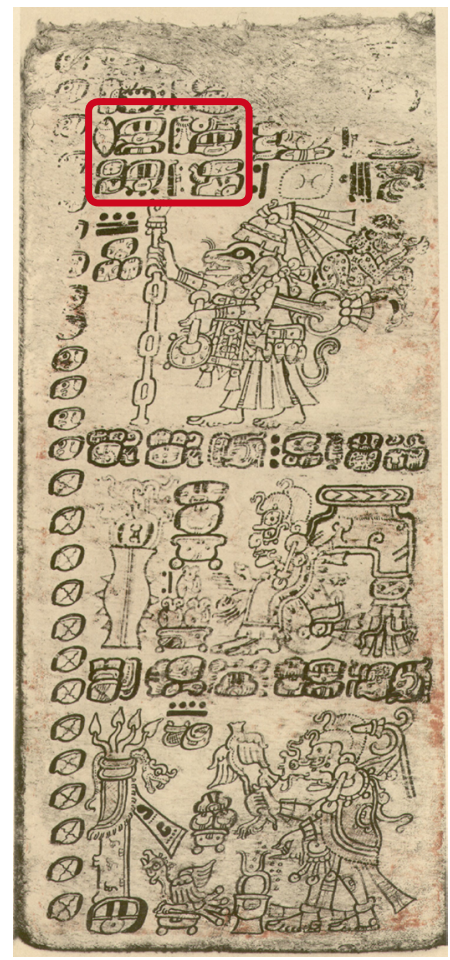

co, bien cimentada por textos escritos desde inicios de la Colonia, indica la presencia de profetas y su continuo deambular por las comunidades de las Tierras Bajas, de manera que podemos sacar como consecuencia que sus profecías bien pueden recoger el estado de ánimo de la comunidad maya, cuando no alentarlo y dirigirlo (Lacadena 2006).

Uno de los papeles religiosos del ajaw, del sagrado señor, del rey, en las cortes del periodo Clásico era interpretar la realidad y escrutar el futuro. Los hallazgos y contextos arqueológicos, la iconografía y los textos epigráficos sitúan a los soberanos mayas haciendo augurios y participando en prácticas que permitían conectar con fuerzas sobrenaturales que les capacitaban para interpretar el devenir. Títulos como $k^{\prime} a$ 'yo'm 'el anunciador' y evidencias de participación en experiencias adivinatorias similares a las mejor conocidas del tiempo de la Conquista recogidas en la Relación de las cosas de Yucatán elaborada por Diego de Landa, como la documentada para un Año Nuevo en Ek' Balam en el Clásico Tardío (Vargas et al. 2007), avalan estas afirmaciones. De otro lado, los profetas son una realidad, al menos para el periodo Postclásico. Unos y otros pretenden adelantarse en el tiempo en la interpretación de la realidad, influyendo mediante determinados recursos y con diferentes legitimidades a las poblaciones a las que van destinados sus augurios y profecías. De modo que tales interpretaciones se pueden considerar una balanza muy aproximada de las crisis y sentimientos de peligro que las sociedades mayas pudieron vivir o, en cualquier caso, angustiarse por ello.

Más aún, los profetas, característicos y frecuentes en las épocas de crisis, no sólo advierten de los males que están por venir, sino que también los justifican desde un punto de vista moral, al presentarlos normalmente como consecuencia o castigo por 
una situación dada, por lo que podemos inferir de los textos proféticos qué situaciones eran consideradas causantes de periodos de inestabilidad:

«Señal que da Dios es que sucederá que muera el rey de esta tierra. Así también que vendrán los antiguos reyes a pelear unos con otros, cuando vayan a entrar los cristianos a esta tierra. Así dará la señal nuestro Padre dios de que vendrán, porque no hay concordia, porque ha pasado mucho la miseria de los hijos de los hijos» (Bricker 1990: 105).

«No había ya buenos sacerdotes que nos enseñaran. Este es el origen del asiento de dos días, del reinado de dos días. Y es también la causa de nuestra muerte. No teníamos buenos sacerdotes, no teníamos sabiduría, y al fin se perdió el valor y la vergüenza. Y todos fueron iguales» (Libro de Chilam Balam de Chumayel 1985: 58).

\section{Crisis políticas}

Pero sin ninguna duda, las situaciones de crisis más recurrentes en el registro documental vienen definidas por los enfrentamientos armados, una situación que parece haberse generalizado también en las diferentes oleadas de colapso que vivieron las sociedades de Petén durante el periodo Preclásico Tardío (Bazy e Inomata 2017), y que se ha documentado de manera sobrada para el denominado «colapso de la sociedad clásica». Junto a las crisis de naturaleza ecológica/económica, los enfrentamientos armados definen mejor que ningún otro proceso la percepción de los momentos de dificultad por parte de los colectivos mayas. La guerra trae consecuencias dramáticas en una amplia variedad de aspectos que se confabulan en la desintegración de las colectividades, acompañada de sufrimiento, dolor y un futuro repleto de sacrificios e incertidumbre.

«Será el estruendo y la confusión de los reyes, y la guerra de Conquista» (Bricker 1990: 159).

«Bajarán escudos, bajarán flechas sobre Chakanputún, en pos de los reyes de la tierra.

(...) Muchas guerras serán hechas por sus moradores» (Bricker 1990: 172-173).

La consecuencia de la guerra es, por una parte, la desaparición del sistema de poder en vigor, y por lo tanto la desarticulación de la sociedad. De otro lado, significa la reaparición de tensiones entre las grandes familias que de manera tradicional han competido por el poder en un dominio determinado, como hemos visto en el texto antes mencionado:

«Señal que da Dios es que sucederá que muera el rey de esta tierra. Así también que vendrán los antiguos reyes a pelear unos con otros, cuando vayan a entrar los cristianos a esta tierra. Así dará la señal nuestro Padre dios de que vendrán, porque no hay concordia, porque ha pasado mucho la miseria de los hijos de los hijos» (Bricker 1990: 105).

La situación adversa de confrontación y de falta de concordia a la que alude el texto es la largamente derivada tras la desaparición de la centralizada Mayapán del paisaje político yucateco en la segunda mitad del siglo XV y el precario y difícil equilibrio político endémico de los nuevos reinos que surgieron tras el vacío de poder producido. El sacerdote maya advierte de la situación de falta de unidad y el peligro evidente que supone la llegada de los españoles justo en ese momento. Pero 
al tiempo presenta la llegada de los españoles como un mandato divino, como un castigo moral:

«así dará la señal nuestro padre Dios de que vendrán, porque no hay concordia» (Bricker 1990: 105).

La pérdida de la articulación política y económica de la comunidad humana originada por la derrota militar de una capital política que lleva implícita la «muerte del rey», viene acompañada además del fin de la articulación ideológica, la desaparición de los grandes sacerdotes que han dado coherencia ideológica y mental a una sociedad, que conocen el pasado y saben emplear los mecanismos para enfrentar el futuro. La confusión social se considera una derivada, pues, de la crisis. Su fin significa también el fin de la sociedad que han alimentado y articulado. Aunque, por intangibles, difíciles de aprehender en el registro material, los mayas asocian de manera recurrente tiempos propicios con la «sabiduría» y «conocimiento» de los líderes políticos y religiosos, y a su vez con la bonanza económica. Bonanza y bienestar económico vienen de la mano de los periodos de buen gobierno, y éste se debe a las buenas cualidades de los propios gobernantes. La ausencia de estas buenas cualidades es causa de la adversidad. El Chilam Balam de Chumayel lo deja claro en este pasaje:

«El Doce Ahau Katun es el octavo katun. Se cuenta en Saclahtun, que es el asiento del katun. (...) De lluvia es la faz de su reinado. (...) Habrá muy buenos Halach uinicques, muy buenos batabes, $\mathrm{y}$ habrá muy buena voluntad en las opiniones de todo el mundo. Se enriquecerán los hombres pobres. Cosechas y cosechas son el hablar del katun, y años ricos y mucha hacienda» (Bricker 1990: 171).

En contraste:

«No hay Gran Conocimiento. Muy perdidos están para ellos el cielo y la tierra. Muy perdida está la vergüenza. Serán ahorcados los soberanos y los reyes de esta tierra, los príncipes de sus pueblos y los sacerdotes de los mayas. Perdido estará entonces el entendimiento y perdida la sabiduría. ¡Preparaos, Itzaes! Vuestros hijos verán extinguirse el Katún a los golpes de la guerra. Loco es el hablar, loco el semblante del reinado del Rey de los espantajos colorados. -Siete son sus platos, siete sus jícaras-. Es la palabra de Dios. Innumerables muertes de horca son la carga de este Katún» (Bricker 1990: 164).

Resulta de singular importancia la coincidencia de estas categorías para explicar la crisis entre los mayas que ocuparon el centro y norte de la península de Yucatán a lo largo de los siglos XVI, XVII y XVIII con algún pasaje que recoge qué pensaron los mayas del periodo Clásico acerca de sus crisis políticas: las escasas veces que lo mencionan lo hacen con tensión evidente por la pérdida de la institución real. Los contados relatos que hemos podido conseguir no sirven tanto para describir colapsos sino para esbozar las concepciones nativas del rompimiento político-social y religioso. Por ejemplo, los problemas que tuvo la dinastía de Palenque frente al insistente acoso militar de la dinastía de Kanu'l (Calakmul) fueron expresados por medio de una retórica altamente ritualizada: el largo texto jeroglífico (Figura 4) que se aloja en el tablero del Templo de las Inscripciones de Palenque registra la historia dinástica de esta capital política en un formato de profecía de $k^{\prime} a t u n$ similar a las profecías de $k^{\prime}$ 'atun recogidas en los Libros del Chilam Balam. En un pasaje asociado con el k'atun 3 Ahaw, que termina en 9.9.0.0.0, se menciona que un rey de Kanu'l atacó Palenque en el año 611 d.C., con consecuencias muy desafortunadas para la dinastía 


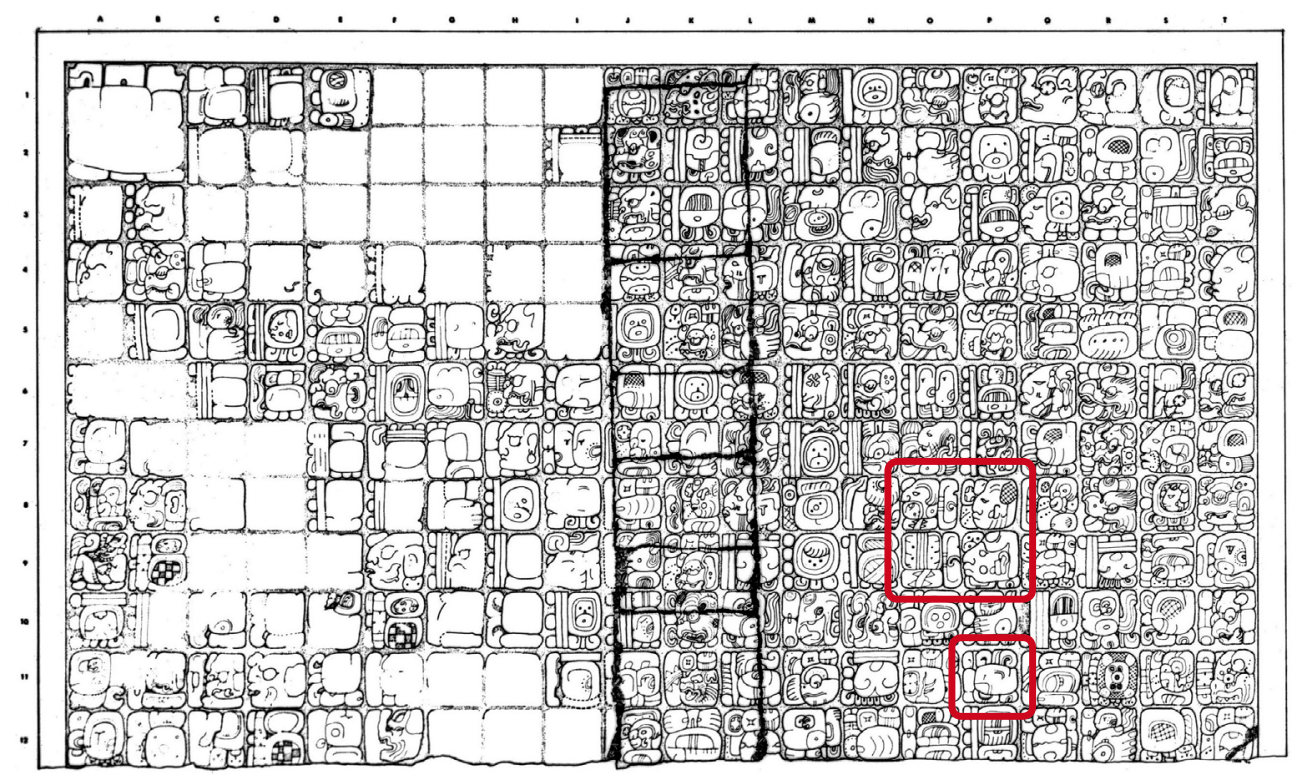

Figura 4. Textos del Tablero Este del Templo de las Inscripciones de Palenque: sataay k'uh[ul] ixik, sataay ajaw, «las sagradas señoras se perdieron, los reyes se perdieron»; ma['] yak'a'w, «no dio [la ofrenda]» (The Linda Schele Drawings Collection, FAMSI; (C) 2000 David Schele).
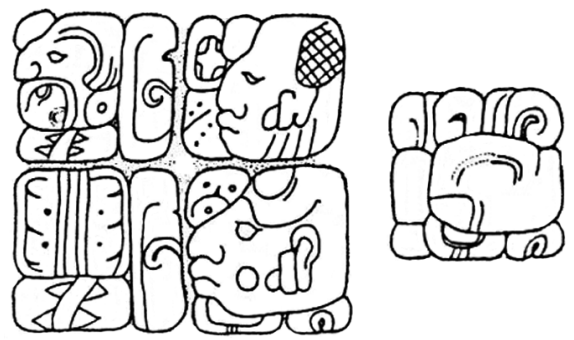

de esta ciudad (Grube 1996; Schele y Mathews 1998: 104-108). Un pasaje subsecuente en la inscripción (posiciones M7-Q9) recoge la siguiente expresión: sataay $k^{\prime}$ 'uhul ixik, sataay ajaw «las sagradas señoras se perdieron, los reyes se perdieron», después de lo cual el texto explicita que hubo una interrupción de los rituales acostumbrados, especialmente en aquellos relacionados con los dioses bajo la tutela de la dinastía y que $m a^{\prime} y a k^{\prime} a^{\prime} w$ «no se dieron» las ofrendas prescritas. Este pasaje de Palenque concuerda con una fórmula bien establecida conocida como «literatura de lamentación», y conecta de manera explícita la dramática adversidad política con la descomposición religiosa ceremonial.

Lo interesante del caso de Palenque es que el propio discurso que une de forma tan clara política y religión se presenta en contexto profético. Las distintas secciones del largo texto que explican lo sucedido en los $k^{\prime}$ atunes consecutivos constan de una primera parte profética y una segunda parte histórica. Los eventos históricos se presentan como una especie de materialización de lo antes descrito en la parte profética, como si fuera algo que debía suceder así. En otro texto (Figura 5), grabado en la Escalera Jeroglífica de la Casa $\mathrm{C}$ del Palacio de Palenque, una nueva referencia a un

\footnotetext{
Iconográficamente, una expresión para la captura de dioses patronos y decadencia de reinos es la captura de palanquines en el campo de batalla. En estas literas decoradas con deidades patronas particulares, fueron transportados los reyes habitualmente, y su captura en el campo de batalla o en la ciudad vencida, significó la captura de la divinidad patrona que patrocinaba a la ciudad y a la dinastía en ella instalada.
} 

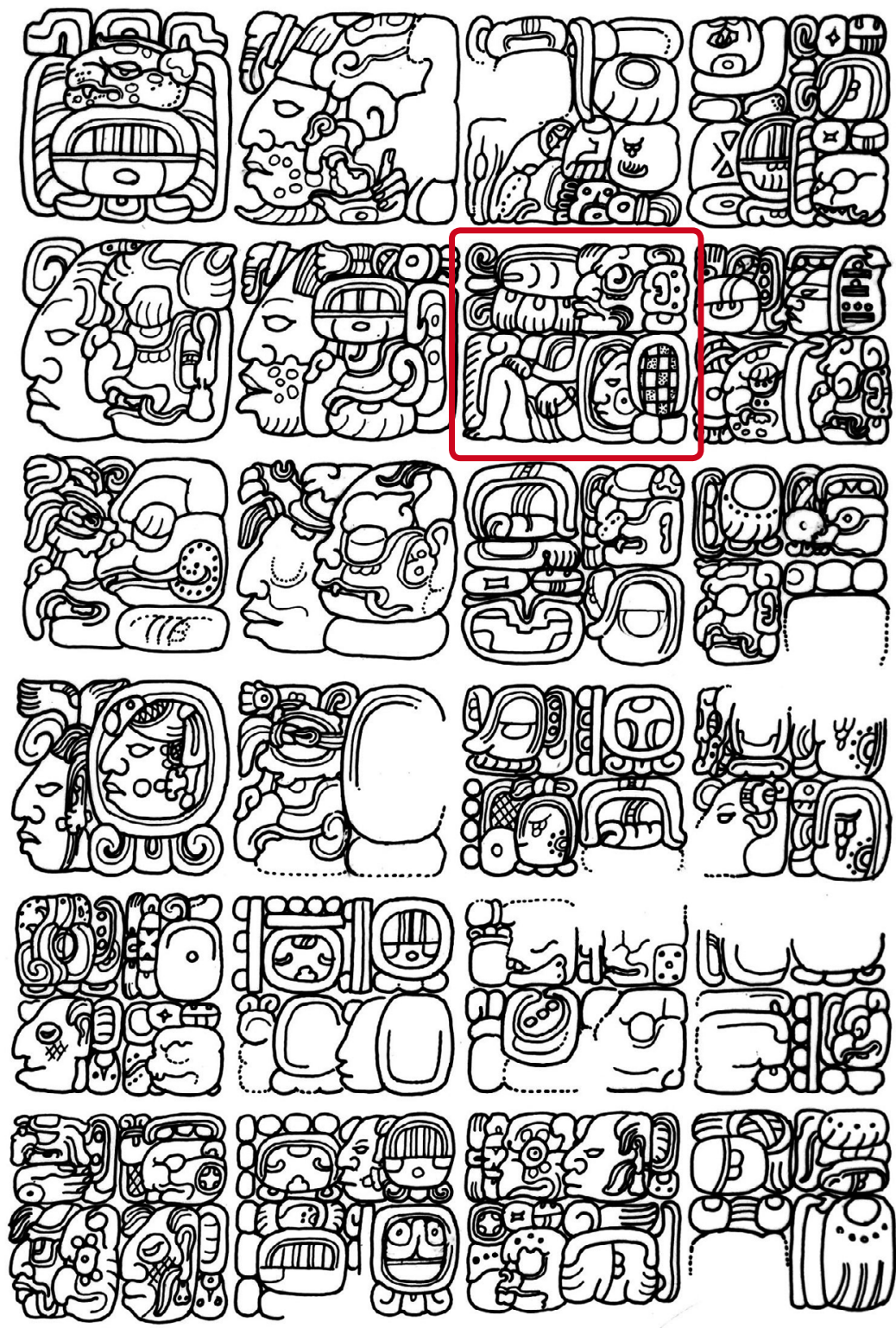

Figura 5. Panel de la alfarda de la Casa C del Palacio, Palenque: ya-le-je GI UNEN-ne

K'AWIL GIII, ya[']lej GI Unen K'awiil GIII, «GI, Unen K'awiil (y) GIII lo han ordenado» (The Linda Schele Drawings Collection, FAMSI; C 2000 David Schele).

ataque exitoso de Kanu'l contra Palenque dice que ya 'lej 'fue ordenado' por los dioses de la Triada de Palenque. Estos textos ciertamente singulares -los gobernantes mayas (como cualquier gobernante en la historia de la humanidad) suelen registrar en sus monumentos a quiénes vencieron, no por quiénes fueron vencidos- señalan la excepcional situación de profunda crisis política que vivió Palenque a finales del 
siglo VI y comienzos del VII, cuando no podía hacer frente a los ataques de Kanu'l y sin duda la dinastía real palencana y su legitimación ideológica eran fuertemente contestadas desde dentro del propio reino. La estrategia de los gobernantes de Palenque fue la de presentar su derrota como la inevitable verificación de una profecía, como la inexorable ejecución de un mandato divino. Los gobernantes no son responsables en realidad del desastre, fue designio de los propios dioses de la ciudad. Este discurso, en realidad, no es muy diferente del mantenido por los profetas mayas del momento de la Conquista cuando llegan a justificar hechos políticos similares como un imperativo sobrenatural, «señal de Dios», que justifica la Conquista.

Sin duda, estamos ante una expresión particular que refiere a un fenómeno local de decadencia de la dinastía en Palenque, pero ofrece un punto de vista nativo de lo que significó este acontecimiento histórico para las Tierras Bajas mayas.

En relatos bastante más tardíos realizados en el Yucatán colonial, las nociones de inestabilidad también están relacionadas con gobernantes efímeros, como indica la expresión cakin xec, cakin ahaulil, «el trono de dos días, el reinado de dos días», del profético Libro del Chilam Balam de Chumayel (Bricker 1990: 600), implicando un reinado de incómoda brevedad. En los tiempos difíciles podía ocurrir una «pérdida de visión» y surgían problemas por el hecho de que no había «ningún gran maestro, ningún gran orador, ningún sacerdote supremo») (minan nohoch can, minan yahau than, minan ahau can), (Bricker 1990: 600, CHU-0586, 058, 0588). La fórmula de Palenque antes mencionada de «las sagradas señoras se perdieron, los reyes se perdieron» encuentra un paralelo casi exacto en el Chilam Balam de Tizimin: ppatbe u cah satai ahaulil, «la comunidad pierde su camino, el reinado se ha perdido» (Edmonson 1982: 105). Este mismo manuscrito también describe el colapso de una ciudad, literalmente, como un ejemplo en el que el gobierno colectivo reemplaza un arreglo más centralizado (uchci puch' tun ich paa tu men u pach tulum, tu men mul tepal), «hace mucho tiempo las piedras de la fortaleza se quebraron, por tomar posesión de los muros, por el gobierno compartido» (Edmonson 1982: 10). En suma, tanto las fuentes epigráficas del periodo Clásico, si bien muy escasas todavía, como las fuentes indígenas del Yucatán de la Colonia reflejan un sistema de categorías en el que la pérdida de los reyes y del liderazgo, y la pérdida de sus sacerdotes equivale a confusión y desorden, es la expresión misma de la confusión y el desorden.

Otra consecuencia explícita en los documentos indígenas coloniales en lo que se refiere al enfrentamiento armado es el abandono de las ciudades como consecuencia de las derrotas bélicas:

«Ocho Ahau. Fue abandonado Chakan-Putún, por los hombres itzaes. Y vinieron a poner sus casas otra vez. Trece dobleces del katun estuvieron establecidos en sus casas de Chakan-Putún. En este mismo Katun fueron los itzaes a vivir bajo los árboles, bajo la ceniza, bajo su miseria» (Bricker 1990: 144).

«Ocho Ahau. Fueron dispersados los itzaes de sus casas por segunda vez, por el pecado de palabra de Hunaceel, por sus alborotos con los itzaes. Trece dobleces del Katun habían estado establecidos, cuando fueron dispersados por Hunaceel, para dar el entendimiento de los Itzaes» (Bricker 1990: 144).

«Trece katunes ejercieron poder. Y fueron traicionados por Hunaceel. Y abandonaron sus tierras. Y fueron a los bosques desiertos que se llaman Tanxulucmul. Cuatro Ahau es el katun en que fue el clamor de las almas. Trece katunes tuvieron bastante con su sufrimiento» (Bricker 1990: 147). 
«Uno Ahau. Fue destruida la comarca de Tancah, nombrada Mayapán. En el primer año tun dentro de Uno Ahau Katun, salió de allí el soberano Tutul y los príncipes de la Tierra y los Cantzuculab. En este Katun fueron vencidos los hombres de Tancah y se dispersaron los príncipes de la Tierra» (Bricker 1990: 48).

El abandono de la capital política, de la ciudad, da una idea de la profundidad de la crisis y el impacto social que ello tuvo en la comunidad. La ciudad es el orden perfecto de la creación, el nivel más complejo de la organización social, política y cultural humana; de manera que su abandono, el irse a «vivir bajo los árboles, bajo la ceniza, bajo su miseria», es pasar de la civilización al desorden, al caos; es la desintegración social y cultural. La pérdida de los reyes que hemos mencionado, la interrupción del ceremonial, la pérdida del conocimiento ${ }^{8}$, la pérdida de la integración sociocultural de la comunidad humana, es expresión fiel de la crisis.

Durante mucho tiempo hemos empleado esta definición de desintegración para explicar el denominado colapso maya Clásico (Webster 2002; Demarest 2013), y expresiones como las mencionadas pueden conectar a la perfección con estas conclusiones a las que hemos llegado los arqueólogos.

A veces, incluso, es abandonado todo un dominio político, y no solo un centro político, lo cual registra situaciones que tienen paralelo con la evidencia de finales del Clásico:

«En el año de mil quinientos cuarenta y uno, fue la primera llegada de los Dzules por el oriente, a Ecab... Quince veintenas de años antes de que llegaran los dzules fue la dispersión de los Itzaes. Fue abandonada la ciudad de Sac-lah-tun, y fue arruinada la ciudad de Kinchil Cobá. Y fue arruinada Chichén ltzá. Y fue abandonada la ciudad que está junto a Uxmal, al sur de la ciudad de Uxmal, nombrada Cib, y también Kabah. Y fue arruinada Seyé, y Pakam, y Homtún, y la ciudad de Tix-calom-kin, y Aké, la de las puertas de piedra. Y fue abandonada la ciudad a donde baja la lluvia del rocío, Etzemal» (Bricker 1990: 51-52).

Los documentos coloniales escritos por conquistadores, frailes y administradores españoles son recurrentes acerca del abandono de los asentamientos y núcleos urbanos por parte de las poblaciones indígenas ante la llegada de las huestes españolas a sus territorios -a veces un abandono momentáneo, a veces destruyendo y quemando todas sus existencias que no pudieron llevarse consigo-, de manera que pudo haber abandonos definitivos. También la destrucción de esos asentamientos por parte de los españoles, o el traslado de poblaciones, pudo significar ese abandono definitivo. Y así, por ejemplo, Breton confirma esta práctica en las Tierras Altas de Guatemala en su estudio del Rabinal Achí:

«¿No devastasteis acaso dos//Tres poblados//Caseríos//Y aldeas: //En Balamwak...?» (Breton 1994: folio 26, 1009; pp. 224-225).

De ello se deduce que la destrucción de los asentamientos, y seguramente de los campos de cultivo, grandes y pequeños, es consecuencia de la guerra.

«En el Ocho Ahau abandonaron sus tierras y se derramaron por todo el país. En Seis Ahau fueron dispersados y acabó su nombre de mayas» (Bricker 1990: 147).

\footnotetext{
8 Si no hay conocimiento, puede resultar lógico el fin de la construcción monumental, de la talla de estelas, de la vida sofisticada en los centros urbanos con grandes construcciones, lujosas tumbas, objetos de elite y demás que, en su grado más dramático, pueden identificar el colapso de la sociedad maya clásica.
} 
Otra expresión de la crisis colectiva que genera el enfrentamiento armado viene definida por la exigencia de retorno que adquieren los vencidos de una ciudad o un territorio respecto de los vencedores. El trabajo colectivo para otro dominio político, para otros señores, se considera en este sentido crítico en el subconsciente maya, y como expresión de su crisis. Tributo es igual a sufrimiento, a merma de los potenciales de producción de una comunidad, y, en consecuencia, de estrés de su trabajo o servicio personal más allá de las exigencias que su sistema productivo o social demandan.

« $¡$ Ay, hermanitos niños, dentro del Once Ahau katun viene el peso del dolor, el rigor de la miseria, y el tributo! Apenas nacéis y ya estáis corcoveando bajo el tributo» (Bricker 1990: 161).

«Solamente por el tiempo loco, por los locos sacerdotes, fue que entró a nosotros la tristeza, que entró a nosotros el 'cristianismo'. Porque los 'muy cristianos' llegaron aquí con el verdadero Dios; pero ése fue el principio de la miseria nuestra, el principio del tributo, el principio de la 'limosna', la causa de que saliera la discordia oculta, el principio de las peleas con armas de fuego, el principio de los atropellos, el principio de los despojos de todo, el principio de la esclavitud por las deudas, el principio de las deudas pegadas a la espalda, el principio de la continua reyerta, el principio del padecimiento» (Bricker 1990: 51).

Asimismo, la derrota militar se considera crítica para la sociedad derrotada, porque una de sus consecuencias, además de las ya listadas, es el sacrificio humano: en el folio 5, Rabinal Achí, hijo del rey de Rabinal Job Toj y comandante de su ejército habla en estos términos a K'iche' Achí, comandante de las fuerzas del rey K'iche' Balam K'iche', el cual ha sido capturado por el primero:

$\begin{array}{ll}X a \text { at-wa-j-im } & \text { que no seas sino comida } \\ \text { Xa } \text { at-per-aj-im } & \text { que no seas sino bocado[s] }\end{array}$

(Breton 1994: folio 5, 150; p.167)

Breton (1994: nota 47, pág. 166) comenta que Rabinal Achí amenaza a su interlocutor con hacerlo carne de sacrificio, el destino reservado a los prisioneros de guerra que eran presentados, como narra el Título Xpantzay II, como alimento de los dioses (Carmack 1981: 134; Coto 1983: 502-503). El sacrificio humano vinculado con la acción militar, si bien muchas veces mostrado bajo la pantalla de un ritual, es un tema frecuente en la iconografía y en la epigrafía del Clásico maya, y, en consecuencia, evidencia momentos críticos para una comunidad.

El Rabinal Achí es explícito incluyendo el siguiente texto que, además de la penuria que impera en la ciudadela fortificada de Job Toj en Rabinal, donde las reservas disminuyen y empiezan a faltar, donde los alimentos están a la vez secos, podridos y producen brotes, menciona:

«Que sólo los grillos//Y las chicharras//Sigan cantando en el interior de la fortaleza//Y de la muralla// Puesto que solo nueve//o diez han regresado a la fortaleza//su muralla// ¡dejemos, pues, de consumir a los blancos niños!» (Breton 1994: folio 8: 255-264; p. 175).

En notas 64 y 66, Breton (1994: 174-175) hace mención a lugares (casas, pueblos) despoblados o desiertos, golpeados por la enfermedad y el hambre, en referencia al estado de desolación en que se encuentran. El consumo de blancos niños refiere a la relación conceptual que existía, para los antiguos K'iche', entre los prisioneros de guerra, el sacrifico y el canibalismo ritual. 


\section{Hacia una caracterización de la crisis como categoría cultural entre los ma- yas de la antigüiedad}

Los documentos insisten sobre problemas concretos de crisis tanto desde un punto de vista de la experiencia vivida como desde la profecía - hechos reales y augurios y profecías- que se combinan para dar forma a la realidad existencial maya.

Los fenómenos de crisis y de resiliencia en la antigüedad maya han venido llamando la atención de la investigación desde hace tiempo, en el primer caso, y más recientemente, en el segundo (McAnany y Yoffee 2009). Los trabajos realizados se han centrado en identificar y describir situaciones o procesos de crisis a partir principalmente del registro arqueológico y epigráfico, evaluando la influencia de aspectos como las sequías, las guerras o la demografía y las formas de responder a su impacto. Nuestro trabajo se orienta, en este contexto, a tratar de indagar en la visión maya de las crisis, desde el supuesto de que el conocimiento de las categorías culturales en torno a ellas tiene, de un lado, valor por sí mismo en el estudio de la cultura y la historia mayas y tiene, de otro lado, valor a la hora de profundizar en la interpretación de episodios de crisis y respuesta sugeridos. ¿Qué consideraba el maya como negativo o no beneficioso para el correcto desarrollo de su mundo? ¿En qué términos lo describía? ¿A qué atribuía su llegada y su desaparición? Para responder a estas preguntas, hemos presentado los primeros resultados del examen de las narraciones que los propios mayas hicieron de estos fenómenos y procesos, atendiendo a cómo los describieron y categorizaron, a través de la revisión de distintas fuentes escritas en lengua maya -libros de Chilam Balam, anales, títulos, etcétera-, que recogen un rico abanico de situaciones que los mayas consideraron malas o negativas en los ámbitos ecológico, económico, social, político y religioso.

La identificación de categorías mayas en relación con las crisis constituye una tarea que tiene valor por sí misma, pues los sistemas de pensamiento mayas forman parte de nuestro objeto de estudio como mayistas. Constituye, asimismo, una aproximación que -como decíamos al comienzo- puede permitirnos avanzar en la comprensión de las crisis que se produjeron en el área maya durante su pasado prehispánico. Obviamente, la explicación contemporánea de los factores que intervinieron en el desencadenamiento de una crisis concreta del pasado ha de basarse en modelos y datos igualmente contemporáneos, pero de ello no se sigue una dicotomía simple entre lo «emic» y lo «etic». En el terreno de la práctica, los factores que tienen existencia propia -independiente del modo en que sean pensados- se entretejen con la interpretación que de ellos hacen quienes los viven para modular las reacciones y respuestas de éstos, las cuales, a su vez, imprimen su huella en los registros arqueológico e histórico. Es a esa interpretación, y desde lo inscrito en una muestra parcial de textos, hacia donde apunta esta revisión.

\section{Referencias}

Armijo Torres, Ricardo. 1999. «Nuevo hallazgo en Comalcalco». Arqueología Mexicana 37: 71-72.

Armijo Torres, Ricardo, Marc U. Zender y Miriam J. Gallegos Gómora. 2000. «La urna funeraria, y el diario de Aj Pakal Tahn sacerdote de Comalcalco durante el Clásico». Temas Antropológicos 22 (2): 312-323. 
Armijo Torres, Ricardo y Marc Zender. 1999. «Una ofrenda conmemorativa del Katun 17 en Comalcalco, Tabasco». Ponencia presentada en Tercera Mesa Redonda de Palenque, 28 de junio a 2 de julio de 1999. Palenque.

Arnauld, M.-Charlotte y Alain Breton, eds. 2013. Millenary Maya Societies: Past Crises and Resilience. Memorias del coloquio international «Sociétés mayas millénaires: crises du passé et résilience», Musée du quai Branly, París, 1-2 de julio, 2011. http://www. mesoweb.com/publications/MMS/.

Bazy, Damien y Takeshi Inomata. 2017. «Multiple Waves of Political Disintegration in the Classic Maya Collapse: New Insights from the Excavation of Group D, Ceibal, Guatemala». Journal of Field Archaeology 42 (2): 82-96.

Breton, Alain, ed. 1994. Rabinal Achí. Un drame dynástique maya du quinzième siécle. Nanterre: Société des Américanistes, Société d'Ethnologie.

Bricker, Victoria. 1990. A Morpheme Concordance of the Book of Chilam Balam of Chumayel. Middle American Research Institute 59. Nueva Orleans: Tulane University.

Carmack, Robert. 1981. The Quiché Mayas of Utatlán: The Evolution of a Highland Guatemala Kingdom. Norman: University of Oklahoma Press.

Códice de Dresde. 1888/1892. Copia digital de las ediciones de Ernst Förstemann. http:// www.famsi.org/mayawriting/codices/pdf/dresden_fors_schele_all.pdf.

Coto, Bernabé. 1983. [Thesavrus verborvn]. Vocabulario de la lengua cakchiquel v[el] guatemalteca, nuevamente hecho y recopilado con summo estudio, trabajo y erudición. Edición de René Acuña. México: Instituto de Investigaciones Filológicas. Universidad Nacional Autónoma de México.

Curtis, Jason H., David A. Hodell y Mark Brenner. 1996. «Climate Variability on the Yucatan Peninsula (Mexico) during the Past 3500 Years, and Implications for Maya Cultural Evolution». Quaternary Research 46: 37-47.

Demarest, Arthur A. 2013. «The Collapse of the Classic Maya Kingdoms of the Southwestern Petén: Implications for the End of Classic Maya Civilization», en Millenary Maya Societies: Past Crises and Resilience, M.-Charlotte Arnauld y Alain Breton, eds., pp. 22-48. www.mesoweb.com/publications/MMS/2_Demarest.pdf.

Douglas, Peter M.J., Mark Brenner y Jason H. Curtis. 2015. «Methods and Future Directions for Paleoclimatology in the Maya Lowlands». Global and Planetary Change 138: 3-24.

Douglas, Peter M.J., Mark Pagani, Marcello A. Canuto, Mark Brenner, David A. Hodell, Timothy I. Eglinton y Jason H. Curtis. 2015. «Drought, Agricultural Adaptation, and Sociopolitical Collapse in the Maya Lowlands». Proceedings of National Academy of Sciences 112 (18): 5607-5612.

Edmonson, Munro S. 1982. The Ancient Future of the Itza: The Book of Chilam Balam of Tizimin. Austin: University of Texas Press.

Gill, Richardson B. 2000. The Great Maya Droughts: Water, Life, and Death. Albuquerque: University of New Mexico Press.

Grube, Nikolai. 1996. «Palenque in the Maya World», en Eigth Palenque Round Table, 1993, Martha J. Macri y Jan McHargue, eds., pp. 1-13. San Francisco: Pre-Columbian Art Research Institute.

Hodell, David A., Jason H. Curtis y Mark Brenner. 1995. «Possible Role of Climate in the Collapse of Classic Maya Civilization». Nature 375: 391-394.

Iannone, Gyle, ed. 2014. The Great Maya Droughts in Cultural Context. Boulder: University of Colorado.

Inomata, Takeshi, Daniela Triadan, Jessica MacLellan, Melissa Burham, Kazuo Aoyama, Juan Manuel Palomo, Hitoshi Yonenobu, Flory Pinzón y Hiroo Nasu. 2017. «High-Pre- 
cision Radiocarbon Dating of Political Collapse and Dynastic Origins at the Maya Site of Ceibal, Guatemala». Proceedings of National Academy of Sciences 114 (6): 1293 1298.

Jobbová, Eva, Christophe Helmke y Andrew Bevan. 2018. «Ritual Responses to Drought: An Examination of Ritual Expressions in Classic Maya Written Sources». Human Ecology 46 (5): 759-781. https://doi.org/10.1007/s10745-018-0019-6.

Kennett, Douglas, Sebastian Breitenbach, Valorie V. Aquino, Yemane Asmerom, Jaime Awe, James Baldini, Patrick Bartlein, Brendan Culleton, Claire Ebert, Christopher Jazwa, Martha J. Macri, Norbet Marwan, Victor Polyak, Keith Prufer, Harriet Ridley, Harald Sodemann, Bruce Winterhalder y Gerald H. Haug. 2012. «Development and Disintegration of Maya Political Systems in Response to Climate Change». Science 338 (6108): 788-791.

Lacadena, Alfonso. 2006. «El origen prehispánico de las profecías katúnicas mayas coloniales: Antecedentes clásicos de las profecías de 12 Ajaw y 10 Ajaw», en Sacred Books, Sacred Languages: Two Thousand Years of Ritual and Religious Maya Literature, Rogelio Valencia y Geneviève Le Fort, eds., 201-225. Acta Mesoamericana 18. Markt Schwaben: Verlag Anton Saurwein.

Libro de Chilam Balam de Chumayel. 1985. Traducción de Antonio Mediz Bolio y prólogo, introducción y notas de Mercedes de la Garza. México: Secretaría de Educación Pública.

McAnany, Patricia A. y Norman Yoffee, eds. 2009. Questioning Collapse: Human Resilience, Ecological Vulnerability, and the Aftermath of Empire. Cambridge: Cambridge University Press.

Schele, Linda y Peter Mathews. 1998. The Code of Kings: The Language of Seven Sacred Maya Temples and Tombs. Nueva York: Scribner.

Turner II, Billie L. y Jeremy A. Sabloff. 2012. «Classic Period collapse of the Central Maya Lowlands: Insights about Human-Environment Relationships for Sustainability». Proceedings of National Academy of Sciences 119 (35): 13908-13914.

Vargas de la Peña, Leticia, Víctor Castillo Borges y Alfonso Lacadena García-Gallo. 2007. «El Mural del Cuarto 22 de Ek’ Balam, Yucatán, México: ritual y profecía de Año Nuevo en el periodo Clásico Maya». Mayab 19: 107-121.

Webster, David. 2002. The Fall of the Ancient Maya: Solving the Mystery of the Maya Collapse. Nueva York: Thames \& Hudson.

Zender, Marc Uwe. 2004. A Study of Classic Maya Priesthood. Tesis Doctoral. Department of Archaeology. University of Calgary. 